\title{
Un épistolier alsacien dans la Grande Guerre
}

Briefe eines elsässischen Artilleristen im Ersten Weltkrieg

Letters of an Alsatian artillerist in the First World War

\section{Françoise Knopper}

\section{OpenEdition}

\section{Journals}

Édition électronique

URL : http://journals.openedition.org/ceg/688

DOI : $10.4000 /$ ceg.688

ISSN : 2605-8359

\section{Éditeur}

Presses Universitaires de Provence

Édition imprimée

Date de publication : 18 novembre 2016

Pagination : 159-169

ISBN : 979-10-320-0087-8

ISSN : 0751-4239

\section{Référence électronique}

Françoise Knopper, «Un épistolier alsacien dans la Grande Guerre », Cahiers d'Études Germaniques [En ligne], 71 | 2016, mis en ligne le 18 mai 2018, consulté le 25 novembre 2020. URL : http:// journals.openedition.org/ceg/688 ; DOI : https://doi.org/10.4000/ceg.688 


\title{
Un épistolier alsacien dans la Grande Guerre
}

\author{
Françoise KNOPPER \\ Université Toulouse Jean-Jaurès, CREG
}

La correspondance de guerre (Feldpost) est un terme générique qui, comme l'article de Rémy Cazals l'expose dans le présent volume, englobe des aspects matériels (gratuité postale, censure, gestion des transports) et des enjeux sociaux, moraux, affectifs. L'intérêt des chercheurs pour un tel corpus n'est pas récent mais les orientations historiographiques qui ont été privilégiées ont évolué ${ }^{1}$. Parallèlement à l'élan que prirent l'histoire des mentalités, l'étude des pratiques quotidiennes et l'histoire des petites gens à la fin des années 1970, l'intérêt se porta sur des carnets de guerre et des journaux intimes de soldats ${ }^{2}$. Un autre tournant a correspondu au visual turn de l'historiographie, lequel a suscité davantage d'intérêt pour les cartes postales imprimées durant la Première Guerre $^{3}$. Puis, depuis les années 2000, avec la prise en compte systématique des ego-documents, ce sont les lettres des particuliers qui ont été collectées par les archives et les musées ${ }^{4}$, celles de gradés comme celles de simples soldats.

Le cas de figure étudié dans cet article s'inscrit dans le prolongement des recherches de Rémy Cazals et peut illustrer le rayonnement qu'exerça MarieLouise Puech en tant que " marraine de guerre ${ }^{5}$ ". En effet, parmi les 550 lettres

1. Un bilan des phases de l'historiographie de la Première Guerre a été établi par Antoine Prost, Jay Winter, Penser la Grande Guerre. Un essai d'historiographie, Paris, Seuil, 2004.

2. Sur la tendance - en Allemagne et en France - à accorder une moindre prépondérance aux seuls événements afin d'analyser aussi les composantes sociales et d'étudier la guerre faite et représentée " d'en bas ", cf. Wolfram Wette (Hrsg.), Der Krieg des kleinen Mannes, eine Militärgeschichte von unten, München, Piper, 1992.

3. Des analyses plus récentes s'inspirent de paramètres de l'histoire culturelle, par exemple: Marie Monique Huss, Histoires de famille. Carte postale et culture de guerre 1914-1918, Paris, Vienot, Paris, 2000 ; Georges Klochendler / Jean-Yves Le Naour, Cartes postales de poilus, Paris, First éditions, 2008; on trouve d'abondantes illustrations, notamment de caricatures, dans Pierre Brouland / Guillaume Doizy, La Grande Guerre des cartes postales, Paris, Hugo-Images, 2013.

4. Pour les fonds allemands, il y a par exemple 100000 documents consultables au Museum für Kommunikation à Berlin: [http://www.museumsstiftung.de/briefsammlung/feldpost-ersterweltkrieg/feldpost.html]. En France, on peut signaler la collection de 65000 cartes à la BDIC (Bibliothèque de documentation internationale contemporaine) : [http://centenaire.org/fr/tresorsdarchives/carte-postale/la-vie-au-front-travers-les-cartes-postales], consultée le 10-01-2016.

5. Cf. Marie-Louise et Jules Puech, Saleté de guerre! Correspondance 1915-1916, Paris, Ampelos, 2015, et Rémy Cazals, 500 témoins de la Grande Guerre, Toulouse, Éditions Midi-Pyrénéennes, 2013, p. 386-388. Les renseignements transmis par R. Cazals montrent que Marie-Louise Puech fut une " marraine de guerre " particulièrement engagée: loin de se limiter à soutenir un seul " filleul " comme la plupart des autres " marraines ", elle a écrit et envoyé des colis à de 
de soldats mobilisés qui lui furent adressées et qui ont été déposées aux Archives Départementales du Tarn, où elles sont en cours d'inventaire, on en trouve une cinquantaine qui sont rédigées en allemand et qui sont toutes dues à un soldat alsacien nommé Auguste Bernard ${ }^{6}$. À partir de quelques indications qu'il donne dans ses lettres et qui sont d'ailleurs résumées par Mme Puech dans un tapuscrit datant sans doute de 1919, nous apprenons qu'A. Bernard était originaire de Saint Jean, près de Saverne, aujourd'hui dans le Bas Rhin. Cet Alsacien - qui dit avoir déjà eu maille à partir avec les autorités allemandes lors de l'affaire de Saverne - avait choisi, à l'âge de 20 ans, de servir le drapeau français; il fut recruté comme artilleur.

Or pour cet Alsacien, les menaces étaient nombreuses. Du côté des tactiques militaires, l'arrière-plan des années 1917-1918 était la grande offensive allemande sur le front occidental, l'emploi du gaz moutarde, le développement de l'aviation ${ }^{7}$. Et concernant le volet plus personnel de ce soldat, son option pour l'armée française le marginalisait non seulement face aux Allemands puisqu'il s'était évadé d'Alsace, mais aussi face aux autres Français puisqu'il était germanophone $^{8}$. Ainsi ses premières lettres de 1917 concernent-elles surtout sa relation à ses parents et le souci qu'il se fait à leur sujet: il expose à Mme Puech qu'il ne peut pas leur donner de ses nouvelles et qu'il redoute par ailleurs que sa famille ne soit confrontée aux pires difficultés face aux autorités allemandes en raison de son départ. Mais, à partir du moment où - à en juger par les lettres - Mme Puech a pu établir un contact avec ses parents, A. Bernard ne parle plus d'eux qu'épisodiquement, signale qu'il reçoit des cartes de leur part, et il lui arrive de prier Mme Puech de leur répondre à sa place.

Proportionnellement, la majorité de sa correspondance date de 1918, lors de la recrudescence des combats, surtout après le 21 mars 1918 ; on note qu'il y a ensuite une reprise de la guerre des tranchées, vers le 6 avril. Après cette date, on voit qu'il fit partie des artilleurs français qui ont mené de durs combats lors des deux dernières tentatives que firent les Allemands pour avancer sur Paris, celle de la fin du mois de mai et du début de juin, puis celle qui se déroula après le 15 juillet 1918 dans la plaine champenoise et aux environs de Reims. Le

nombreux soldats ayant des difficultés (prisonniers de guerre, soldats dont la famille était restée en pays envahi, camarades que son mari savait être de famille pauvre). Une partie de l'argent provenait de ses relations canadiennes et de Jane Addams, féministe et pacifiste américaine.

6. A. Bernard fait partie des auteurs présentés par Magalie Amiel, Paroles de poilus. Lettres reçues par Marie-Louise Puech-Milhau pendant la Grande Guerre, mémoire de maîtrise, Toulouse-II Le Mirail, 2001.

7. Cf. Volker Berghahn, Der Erste Weltkrieg, München, Beck, 2003, p. 96 et suivantes.

8. Des chiffres sont donnés par Pierre Montagnon, Dictionnaire de la Grande Guerre, Paris, Pygmalion, 2013, p. 38 : « 280000 Alsaciens-Lorrains portèrent l'uniforme allemand et autour de 20000 combattirent dans l'armée française ". Gerhard Hirschfeld / Gerd Krumeich / Irina Renz, Enzyklopädie Erster Weltkrieg, Paderborn, Schöningh, 2009, p. 454-456, indiquent que, dans l'armée française, 12000 soldats avaient un passeport allemand. Sur la place d'Alsaciens et Lorrains dans la Première Guerre, côté français et allemand, nous renvoyons aussi aux travaux de François Roth, notamment à Alsace-Lorraine, Histoire d'un "pays perdu ", de 1870 à nos jours, Nancy, éditions de la Place Stanislas, 2010, ou de Jean-Noël Grandhomme / Francis Grandhomme, Les Alsaciens-Lorrains dans la Grande Guerre, Strasbourg, La Nuée bleue, 2013. 
terminus ad quem de cette correspondance fait quant à lui assister à la façon dont A. Bernard sortit de la Grande Guerre et géra le processus de démobilisation ${ }^{9}$ : conséquent dans son option patriotique et soucieux de donner une nouvelle preuve de sa bonne foi ${ }^{10}$, il garda l'uniforme et s'engagea dès octobre 1918 pour aller en Algérie, à Constantine ${ }^{11}$.

Comme elle le fit pour les nombreux soldats avec lesquels elle entretint une correspondance pendant la guerre, M.-L. Puech a conservé tous les documents envoyés par A. Bernard, enveloppes comprises, bien qu'il s'agisse, comme nous allons le voir, de textes dont le modeste contenu ne faisait pas découvrir d'informations inédites ou de secrets sensationnels. Si leur archivage a néanmoins requis l'attention d'une destinataire aussi expérimentée qu'elle le fut, il semble opportun que les chercheurs les regardent de près et les insèrent dans la vaste littérature des " poilus ". Après avoir rappelé quelques problèmes de méthode que ce genre de corpus épistolaire peut poser, nous nous consacrerons au statut de l'écrivain non professionnel que fut A. Bernard, puis nous étudierons les manifestations et les répercussions de son militantisme patriotique.

\section{Respect d'usages épistolaires}

L'institution des " marraines de guerre " fut pensée initialement en janvier $1915^{12}$; c'est leur esprit bénévole et bienpensant qui fut appliqué par M.-L. Puech. Il était demandé d'apporter un soutien psychologique et parfois matériel aux soldats qui étaient particulièrement démunis, soit parce qu'ils souffraient d'isolement soit parce que leur solde ne suffisait pas à couvrir leurs besoins ${ }^{13}$. Tel était le cas d'Auguste Bernard, à qui M.-L. Puech a envoyé courriers et mandats. Toutes les lettres prouvent à quel point A. Bernard a su apprécier cette amitié de papier qui lui était offerte et a exprimé sa reconnaissance, de façon respectueuse, scrupuleuse, même si ses termes paraissent maladroits. Ses missives commencent par "liebe gute Dame ", puis par un remerciement pour la réception de la lettre précédente et, toujours, par le souhait que Mme et M. Puech soient en bonne santé. La signature ne varie jamais : "Euer treuer Elsässer Auguste Bernard ".

9. Sur la complexité de ce processus, cf. l'article synthétique de Bruno Cabanes, " Démobilisation et retour des hommes ", in Stéphane Audouin-Rouzeau / Jean-Jacques Becker, Encyclopédie de la Grande Guerre, Paris, Perrin, 2012, t. 2, p. 679-700.

10. Le 4 octobre 1918, A. Bernard résume sa conversation avec le Général qui lui déconseille de partir pour l'Algérie.

11. John Horne in "Démobilisations culturelles après la Grande Guerre ", 14-18 Aujourd'hui Today - Heute, (2002) 5, p. 45-53. Et Jacques Frémeaux / Michèle Battesti, Sortir de la guerre, Paris, Presses de l'université Paris-Sorbonne, 2014.

12. Henriette de Vismes, Histoire authentique et touchante des marraines et des filleuls de guerre, Paris, Perrin, 1918.

13. Cette institution devint populaire et quelque peu dévoyée, cf. Jean-Yves Le Naour, " Marraines de guerre ", in Dictionnaire de la Grande guerre, Paris, Larousse, 2008, ainsi que sa description des fantasmes qu'elles suscitaient dans Le Naour, Misères et tourments de la chair durant la Grande Guerre. Les mœurs sexuelles des Français, 1914-1918, Paris, Aubier, 2002, p. 70 et suivantes. 
Mais la correspondance en temps de guerre était soumise à des contraintes extérieures qu'il importe de ne pas sous-estimer. À commencer par le fait que, si l'artilleur A. Bernard prend la plume aussi fréquemment, cela résulte en partie d'une stimulation officielle: la rédaction de courriers était en effet à la fois contrôlée ${ }^{14}$ et encouragée par les autorités militaires, qui y voyaient un moyen de maintenir le moral des troupes, en France comme en Allemagne ${ }^{15}$, de sorte que les courriers bénéficiaient de la franchise postale des armées ${ }^{16}$.

Ensuite, le début de ses lettres, qui contiennent invariablement la formule " ich bin gesund und munter ", correspondait de manière générale à la civilité attendue dans une correspondance de guerre ${ }^{17}$. L'usage - précisément encouragé par les instances militaires - était de rassurer le front de l'arrière, les civils, les familles et amis. Sous la plume d'A. Bernard, cela permettait en outre de garder un lien avec la société civile puisque - et cela vaut absolument pour chacune de ces occurrences - il s'enquiert aussi de la santé de Mme Puech et de son mari: de tels égards pour autrui faisaient perdurer le lien social. Il arrive souvent qu'il écrive un billet simplement pour dire qu'il est " en bonne santé physique et morale " (" gesund und munter ") et qu'il en espère autant pour Mme Puech et son mari. De tels billets témoignent d'un besoin existentiel d'entretenir un contact, même minime, avec la société civile. Et Mme Puech veillait à entretenir cet intérêt pour les civils puisque, dans une lettre du 26 octobre 1918 - précieux document car nous n'avons pas trouvé dans ce fonds d'autre missive écrite par elle à ce soldat -, rédigée dans un allemand parfait, elle informe A. Bernard des ravages que causait la grippe à Paris.

Un autre problème que peut soulever le maintien de telles formes de civilité épistolaire dans ce contexte militaire particulier est de savoir s'il serait licite d'aller jusqu'à utiliser la formule de " culture de guerre ${ }^{18}$ " ou inversement de signaler la persistance d'une sociabilité de paix, d'une " culture de paix ". En somme d'examiner si, pour reprendre une interrogation que les historiens des mentalités posent souvent, ce serait dans le cadre d'une " culture " spécifique à la Première Guerre que ce soldat a réussi à résister à tous les chocs - physiques et émotionnels - malgré la dureté de ses conditions de vie, et ce sans pour autant perdre le sens des usages et sans étouffer sa sensibilité.

14. Sur la censure, cf. les travaux de Rémy Cazals cités dans le présent volume. On trouve par exemple dans la correspondance d'A. Bernard la lettre R. pour Reims ou Com. pour Compiègne, néanmoins il cite aussi quelques noms de lieux, sans doute quand il est permissionnaire.

15. Bernd Ulrich, " Die Kriegskorrespondenz im militärischen Kontext - Beschwerden, Stimmungsberichte und Zensur ", online: [http://www.erster-weltkrieg.clio-online.de/_Rainbow/ documents/Augenzeugen/ulrich2.pdf], consulté le 18-12-2015.

16. Le service postal des armées qui s'était développé lors de la guerre de 1870 connut une forte extension durant la Première Guerre puisque le nombre des mobilisés était devenu beaucoup plus important.

17. Jürgen Ruby (Hrsg.), Es geht mir gut. Deutsche Feldpost von 1879 bis 2010, Ausstellungskatalog, Berlin, Militärhistorisches Museum der Bundeswehr, 2011.

18. C'est une approche à laquelle souscrit par exemple sans réserve Benoît Amez, Dans les tranchées : les écrits non publiés des combattants belges de la Première guerre mondiale - Analyse de leurs expériences de guerre et des facteurs de résistance, Paris, Publibook, 2009, p. 20. 
Dans le cas d'A. Bernard, des arguments plaident en faveur de la complémentarité des deux notions. D’une part, comme c'est par horreur de l'ennemi que ce soldat alsacien s'est engagé, on peut déceler chez lui une forme de " consentement " à la violence et à la guerre qui constitue, selon l'Ecole de Péronne, le socle de la " culture de guerre ${ }^{19}$ ". Et son intériorisation du combat semble se confirmer quand il prend la décision, début octobre 1918, de garder l'uniforme. Cependant, nous inclinons à abandonner l'emploi de ce terme de " culture de guerre " et à déceler dans ces lettres d'A. Bernard une " culture de paix $^{20}$ ", à savoir des pratiques qu'il savait usitées en tant de paix dans la mesure où, comme cela vient d'être mentionné, il reste proche de sa famille, cherche le dialogue avec des amis, conserve des formes de sociabilité même dans les tranchées.

Cette complémentarité - prédisposition à la guerre et attachement à des usages de paix - a sans doute aussi été amplifiée chez A. Bernard par les influences exogènes - celles d'autorités militaires - qui avaient l'objectif de renforcer l'endurance et sont omniprésentes dans cette correspondance.

\section{Statut d'un écrivain ${ }^{21}$ non professionnel}

Les recueils imprimés de documents contenant des lettres de mobilisés sélectionnent de préférence des lettres d'étudiants, d'artistes ou de jeunes gens qui appartenaient à des milieux bourgeois et avaient la pratique du raisonnement et de l'écriture. Or un des intérêts présentés par les lettres d'A. Bernard résulte au contraire de ses origines rurales. C'est un épistolier qui peut se ranger dans la catégorie des écrivains occasionnels, qualifiés d'" ordinaires " et étudiés sous l'impulsion de Roger Chartier ${ }^{22}$.

En effet, les lettres sont toutes de sa main, sa manière de calligraphier les caractères gothiques est régulière, sans rature, son orthographe et sa grammaire sont relativement correctes, malgré son inexpérience en la matière ${ }^{23}$. En

19. Stéphane Audoin-Rouzeau / Annette Becker, 14-18, retrouver la guerre, Paris, Gallimard, 2000 ; id., "Violence et consentement: la 'culture de guerre' du premier conflit mondial ", dans Jean-Pierre Rioux/ Jean-François Sirinelli (dir.), Pour une histoire culturelle, Paris, Seuil, 1997, p. 251-271. L'historiographie actuelle insiste sur la complémentarité de leurs points de vue avec ceux, opposés, de Rémy Cazals et Frédéric Rousseau.

20. Frédéric Rousseau et alii (dir.), Guerres, paix et sociétés, 1911-1946, Neuilly, Atlande, 2004, p. 667-674.

21. Sur la différence avec les écrivains de métier, cf. Nicolas Beaupré, Écrire en guerre, écrire la guerre, France, Allemagne 1914-1920, Paris, CNRS éditions, 2006. Mais le risque d'imprécision du terme d'“ écrivains combattants " a été montré dans les comptes rendus de R. Cazals et F. Rousseau [http://www.crid1418.org/bibliographie/commentaires/beaupre_rousseau.html], consulté le 15-01-2016).

22. Roger Chartier et alii (dir.), La correspondance. Les usages de la lettre au XIXe siècle, Paris, Fayard, 1991.

23. Montagnon, Dictionnaire de la Grande Guerre, p. 496, formule le même jugement à partir de lettres de paysans francophones: "Par-delà les renseignements sur l'existence du poilu et les espoirs d'un blessé, la lecture des originaux surprend. La calligraphie, l'orthographe de ces 
revanche, l'expression est celle d'un jeune homme qui - tout en respectant les règles - n'a pas de bagage littéraire : le vocabulaire est réduit, répétitif, la syntaxe est rudimentaire, les signes de ponctuation et les majuscules sont rares, et les formules - y compris celles de politesse - sont stylisées. Souvent le code oral l'emporte sur le code écrit. Les missives sont courtes, on a fréquemment affaire à quelques lignes inscrites sur un petit morceau de mauvais papier ou sur une carte de correspondance du service des armées.

Le courrier de remerciement que ses parents adresseront à Mme Puech à la fin de la guerre, est syntaxiquement plus abouti mais de ce fait aussi plus impersonnel ; à cela s'ajoute le fait que leur vision de la guerre est globale et rétrospective. Ce sont des remerciements sincères mais conventionnels, signés par des parents qui partagent l'euphorie des Alsaciens à la fin de l'année 1918 et qui expriment leur reconnaissance pour la générosité de Mme Puech. Ce côté apprêté, que nous ne trouvons pas chez leur fils, ressort par exemple de leur lettre du 8 décembre 1918: ils disent vouloir exprimer leur gratitude pour le soutien de Mme Puech " durant les quatre années de guerre ", alors qu'il est notoire que les marraines de guerre n'existaient que depuis 1915 et que, de toute façon, la correspondance entre Mme Puech et A. Bernard avait commencé assez tardivement.

Comme beaucoup de jeunes Alsaciens ${ }^{24}$, Bernard ne maîtrisait que très mal la langue française, bien qu'il ait plusieurs fois fait l'effort d'écrire en français à M. Puech, lequel ne savait pas l'allemand; il annonce en 1919, après la démobilisation, qu'il s'emploie à perfectionner sa pratique du français. Ses gallicismes sont rares mais on en trouve, par exemple: "viele sind krank gefallen ", " heute ist es gut warm " (11 avril 1918). L'emploi de ihr et euch, là où des formules de politesse seraient attendues (d'autant que Mme Puech vouvoie A. Bernard), est probablement dialectal.

Ces lettres sont bien structurées, presque trop peut-on dire, car, si elles sont courtes et écrites à la hâte, leur schéma en devient squelettique: compliments introductifs, remerciements, assurance de la bonne santé, et indication que la lettre est finie. Pour en donner un exemple, la lettre du 14 avril 1918 (qui correspond à la reprise de la guerre des tranchées depuis dix jours) :

liebe gute Dame!

Ich will euch mitteilen, daß ich noch gesund und munter bin, was ich auch von euch hoffe. da wo wir sind ist es noch immer ruhig, aber man weiß nicht ob es noch lange so ist. So will ich mein schreiben schließen mit vielen grüßen an euch und euren Gatten.

garçons, gens simples, sont bonnes voire excellentes. L'école primaire avait parfaitement rempli son travail, sans parler du patriotisme qu'elle avait inculqué. "

24. Le cas de Dominik Richert correspond au parcours, inverse, d'un Alsacien qui préféra, du fait de sa méconnaissance du français, rester du côté allemand, mais sans se rallier à la propagande du Reich, cf. Hilda Inderwildi, "Naissance et construction d'une conscience pacifiste dans les rangs des paysans alsaciens au moment de la Première Guerre. L'exemple de Dominik Richert (18931977) ", in Jean-Paul Cahn et alii (dir.), De la Guerre juste à la paix juste: Aspects confessionnels de la construction de la paix dans l'espace franco-allemand, Villeneuve d'Ascq, Septentrion, 2008, p. 199-210. Cf. aussi la rubrique qui lui est consacrée par Cazals, 500 témoins, p. 393-395. 
Euer treuer Elsässer Auguste Bernard.

Es gibt keinen Urlaub mehr.

On ne trouve dans ces lettres ni abstractions ni symboles (sauf deux modestes fleurs séchées) mais il arrive que des expressions imagées voire populaires soient insérées. Le rire est alors malicieux, brièvement rabelaisien, que ce soit pour dire à quel point l'administration des Allemands en Alsace est " pénible " (" es ist ein Kreuz [mit den Deutschen] ") ou pour commenter l'échec de l'offensive que les Allemands avaient tentée en avril 1918: "Die Deutschen glaubten sie sind für Ostern in Paris, das gibt es nicht mehr. Hindenburg wird sich hinten an den Ohren kratzen " (12 avril 1918).

La pudeur dans l'expression des souffrances est une autre constante dans les lettres d'A. Bernard. D'aucuns pourraient suggérer qu'une telle retenue est probablement renforcée et par la peur de la censure et par la pauvreté du champ d'expression. Mais ces explications ne nous suffiraient pas car A. Bernard ne se départit jamais de cette pudeur. Il remercie pour l'argent envoyé, promet de rembourser plus tard, signale parfois un emploi qu'il a trouvé dans une usine durant sa permission, et sa précarité s'aggrave après la démobilisation, à preuve les trois télégrammes demandant " ENVOI URGENT MANDAT ». Mais jamais il n'accuse le système, alors qu'il était notoire que la solde ne suffisait pas si un soldat n'avait pas d'autres ressources ${ }^{25}$.

Autre marque de pudeur: il se borne à annoncer que les soldats vont retourner dans les tranchées, sans faire allusion au travail, en fait très pénible, de terrassement. Ou bien il signale les intempéries mais il ne parle de maladies que rarement et tardivement, et ce uniquement à propos de ses compagnons, pas à propos de sa propre personne. Il n'évoque la mort, la sienne et celle de ses camarades, qu'une fois, dans la lettre du 13 août 1918, qui est à cet égard exceptionnelle: A. Bernard signale qu'il a échappé de justesse à la mort et, comme il le répète, il est très affecté par le fait que son sous-officier ainsi que tous ses camarades ont été tués, qu'il y a beaucoup de blessés et que son régiment n'a plus de canon.

Pour le lecteur, ce texte du 13 août est saisissant à plusieurs titres. D'un côté en raison de la date qui se situe après la seconde bataille de la Marne et à une période où il devenait en fait évident que la guerre allait être gagnée par la France et ses alliés. Et d'un autre côté, sur le plan de la sensibilité de l'auteur, en raison de la sobriété de ton qu'il réussit à conserver: sans nier la dureté de la guerre, il continue à marquer que le goût de vivre et le sens - à la fois collectif et personnel - à donner au combat sont les deux valeurs qui devraient l'emporter.

25. Amez, p. 189. 


\section{Militantisme patriotique}

Le patriotisme, qui se manifeste en continu dans ces lettres, est sans nul doute la clef de l'endurance et du courage de ce soldat alsacien. Il formule explicitement son attachement (" mein geliebtes Frankreich"), respecte la hiérarchie militaire (" mein Unteroffizier ", " heute hat der General mit mir gesprochen "). En juillet 1918, il aspire au moment où le drapeau tricolore flottera en Alsace: ils y seront alors " heureux comme jamais " (" glücklich wie noch nie "), mais, indiquant à M. Puech à quel intermédiaire son courrier sera à expédier à Paris, il estime qu'il sera préférable de ne pas préciser qu'il a choisi de servir la patrie française.

Son horreur de l'ennemi, qu'il désigne par "Deutsche Hunde ", parfois par "Preußen ", avait motivé son départ de l'Alsace et elle ne disparaît à aucun instant. L'intention est assurément de venger la défaite de 1870, laquelle avait notoirement renforcé le sentiment identitaire en Alsace et en Lorraine. Chez A. Bernard, il serait cependant incomplet de s'en tenir à l'impression que cette haine se résumerait à son identité alsacienne ${ }^{26}$ et à la veine des dessins de Hansi, car c'est en soldat qu'il la met en action, si bien que sa perception de la Grande Guerre est celle d'une guerre " juste ». Toutes ses lettres, dès 1917, montrent qu'il estime que c'est du côté français que cette guerre est une guerre défensive: c'est ce qui ressort du fait qu'il est beaucoup plus souvent question d'attaques allemandes ("Angriffe der Deutschen "), comme si les troupes françaises ne faisaient que répliquer. La mort de soldats allemands (lettre du 3 juillet 1918) ou la capture de prisonniers ne lui inspirent aucune pitié.

Une lettre du 23 juin 1918, qui est envoyée de Verberie dans l'Oise et annonce qu'il n'y aura plus de permission, traduit encore une fois cette perception de la guerre " juste " :

Heute ist Sonntag, aber leider kann ich nicht zu euch kommen, ihr seht ja die Karte und wir sind ungefähr 25 Kilo. von den Schützengräben, aber wir [...] erwarten mit gutem Vertrauen auf einen großen Angriff der Deutschen. In diesen schweren Tagen wo mein liebes Frankreich von dem BOCH (sic) bedroht ist darf man das Vertrauen nicht aufgeben. Bei mir zu Hause sagt man das Segenswort ${ }^{27}$.

26. Des nuances sont actuellement apportées à la thèse selon laquelle la propagande wilhelminienne aurait échoué en Alsace. Sur cette divergence entre historiens à propos de la propagande allemande en Alsace sous Guillaume II, cf. B. Ulrich, "Kriegskorrespondenz » : " [Hiery vertritt] eine dezidiert andere, auf Wahlanalysen beruhende These als Wehler im Hinblick auf die Integration des Reichslandes in das Reichsgebiet: Hiery vermutet die Elsaß-Lothringer unmittelbar vor dem Krieg auf dem sicheren Weg hin zu loyalen deutschen Staatsbürgern " (Hermann Hiery, Reichstagswahlen im Reichsland. Ein Beitrag zur Landesgeschichte von Elsaß-Lothringen und zur Wahlgeschichte des Deutschen Reiches 1871-1918, Düsseldorf, Droste, 1986, p. 371).

27. Il s'agit de la formule usuelle qui clôt un culte protestant et repose sur l'Ancien Testament en se référant à la bénédiction accordée par Dieu :

Und der HERR redete mit Mose und sprach: Sage Aaron und seinen Söhnen und sprich: So sollt ihr sagen zu den Israeliten, wenn ihr sie segnet:

Der HERR segne dich und behüte dich;

der HERR lasse sein Angesicht leuchten über dir und sei dir gnädig; der HERR hebe sein Angesicht über dich und gebe dir Frieden. 
Il se glisse dans ce courrier deux termes nouveaux chez lui, celui de " confiance " ("Vertrauen "), qui figurera encore dans une lettre suivante, et de " bénédiction " ("Segen "). Ces deux termes font écho à d'autres allusions qui sont faites par A. Bernard, durant cette même période, au moment où la guerre sera finie (" fertig "). Dans toute sa correspondance il n'y avait d'ailleurs pas eu une once de défaitisme: l'ensemble est traversé par la croyance en la victoire de la France, et cela aussi faisait partie des éléments structurant du patriotisme de ce soldat. Mais cette croyance en la victoire n'était jusqu'ici pas explicitée et elle ressortait principalement de son énergie indéfectible, alors que, en ce mois de juin 1918, la devise est énoncée : «il ne faut pas perdre confiance ». Nous décelons ici un signe de lassitude, celle des soldats sur le front et peut-être celle que notre épistolier prête aussi, à juste titre, au front de l'arrière, lassitude que la foi en Dieu - par ailleurs rarement mentionnée par A. Bernard - aiderait à surmonter. Peut-être encore les autorités militaires avaient-elles pour leur part préparé les soldats à l'offensive allemande de l'été - celle qui sera si meurtrière pour le régiment d'A. Bernard - et avancé ce terme-clé qu'il traduit dans cette veine chrétienne par "Vertrauen".

Le patriotisme d'A. Bernard se manifeste également par un autre biais, celui du choix de ses cartes postales: point de caricatures, mais des photographies en noir et blanc, des " cartes-vues ${ }^{28}$ ", qu'il pouvait acheter dans les endroits où il passait ou stationnait, dans l'Aisne, la Seine Inférieure, l'Oise, la Champagne, plus tard de Strasbourg, et montrant souvent des bâtiments ou des rues bordées de maisons en ruines. L'interprétation de ce genre de cartes postales en temps de guerre est complexe puisqu'il faut veiller aujourd'hui à ne pas utiliser de critères anachroniques. Pour notre part, du fait que la plupart des lettres d'A. Bernard sont concises, il nous semble que ses cartes témoignent de la même sobriété mais qu'elles correspondent en outre à sa conception patriotique de la guerre. En effet, le choix de telles photographies n'est pas caractéristique de la palette des cartes postales qui étaient alors à disposition: selon des spécialistes de la question, par exemple Christophe Doisy ou Katrin Kilian déjà cités, la majorité des cartes achetées par les soldats - ou du moins mises à leur disposition - représentaient plutôt soit des personnages auréolés de prestige (souvent les grands généraux ou des groupes de soldats), soit des caricatures de l'ennemi, soit encore des décors mièvres avec dictons enamourés. Or la vision transmise par A. Bernard, si on la déduit de ses cartes et de sa correspondance privée, transmet l'atmosphère du combat, sans glorifier les acteurs et sans embellir la guerre - il ne s'agit pas pour lui de romantiser la représentation de ruines -, mais en soulignant son entière acceptation de la mission patriotique à remplir. En somme, le choix de telles cartes postales suggère qu'il faudrait, selon lui, rudement combattre et traverser des champs de ruines pour résister à l'ennemi et rétablir paix et liberté. Il est significatif, que de passage à Strasbourg le 12 janvier 1919, il ait choisi

Denn ihr sollt meinen Namen auf die Israeliten legen, dass ich sie segne. (4. Buch Mose 6, 22-27).

28. Brouland / Droizy, La Grande Guerre des cartes postales, p. 10-11. 
d'envoyer une carte représentant le Palais de l'université de Strasbourg et ait écrit au verso en français, pour transposer sa représentation du triomphe et de la prééminence de la civilisation française.

L'envoi de telles cartes à Mme Puech indique en outre qu'il avait conscience, peut-être à son insu, que cette guerre marquerait durablement l'histoire francoallemande et qu'il était bon d'en apporter des preuves matérielles: à savoir des preuves non seulement des endroits qui furent stratégiques mais aussi de sa présence individuelle dans ce grand ensemble historique et militaire. Il ne s'agit pas seulement de dénoncer la violence de l'ennemi et l'ampleur des destructions, il s'agit de donner un aperçu du poids historique de l'instant vécu. Ces cartes postales élèvent ainsi cette correspondance privée - et ténue - au niveau supérieur de l'imprimé.

Il est indéniable qu'il y avait aussi, chez lui comme chez d'autres jeunes soldats, une curiosité touristique, puisque la mobilité engendrée par la guerre et les permissions lui faisaient découvrir des villes et des régions (Soissons, Reims, Paris, Marseille). Il est enfın aussi envisageable que ce soit une de ses manières de manifester son amitié pour autrui et qu'il ait cherché des motifs susceptibles de convenir aux Puech, comme son choix d'une carte montrant l'église réformée de Paris, clin d'œil nous prouvant l'attention qu'il savait porter à leurs sentiments religieux.

Autrement dit, c'est à la même période que deux tendances se recoupent chez cet épistolier : il fait l'expérience d'une action collective et d'enjeux graves, et il s'initie à une communication privée qui lui apprend à gérer ses soucis intimes. La perception de la guerre par ce soldat a d'abord éveillé en lui un besoin de dialoguer et de tisser des liens affectifs avec la société civile, d'abord avec ses parents, puis avec le couple Puech qui lui apporte de l'aide psychologique et financière. De plus, le domaine du privé recoupant étroitement le domaine de la collectivité, les lettres commencent et se terminent par " $i c h$ " mais le " wir " qui désigne les autres soldats de son régiment n'est jamais absent. Comme l'écrit R. Cazals, une correspondance en temps de guerre, même adressée à un destinataire précis, a un enjeu public.

Bien que leur contenu n'apporte pas de connaissances nouvelles en matière de tactique et que les stratégies d'écriture y soient peu variées, les missives d'A. Bernard indiquent l'importance qu'une telle correspondance avait pour un soldat, d'abord pour dire, et donc aussi se dire, que la valeur première, essentielle, était celle de rester en vie. Cet échange illustre également plus globalement certains aspects que pouvaient prendre les relations entre militaires et civils durant la Grande Guerre: les soldats comme A. Bernard ne se bornaient pas à relayer une propagande belliciste, ils assumaient délibérément ce qu'ils estimaient relever de leur devoir et, sans se départir du respect filial et de leur attachement identitaire à leur petite patrie, ils choisissaient de donner un sens à leur existence en combattant pour l'État.

Enfin, cette correspondance nous en apprend - indirectement - beaucoup sur l'engagement idéologique et social du couple Puech. Si Jules Puech avait d'abord 
été mobilisé puis avait travaillé dans le service auxiliaire, son épouse faisait partie des civils qui veillaient à se tenir au courant de ce qui se passait sur le front. Tous deux ont entrepris des actions pour faciliter le sort des soldats par les mandats ou, comme le montrent les remerciements qu'A. Bernard adresse à M. Puech, par l'obtention de permissions ou de travail rémunéré. Vu son attention indéfectible portée au sort d'autrui, nous sommes heureuse de rendre à notre tour hommage au rayonnement de M.-L. Puech; sa personnalité et son engagement viennent enrichir le tableau - déjà largement exploré - du rôle économique, civique et social des femmes dans la Première Guerre. Il est à noter que, si la complexité de cette question commence à être bien démontrée ${ }^{29}$, nous n'avons en revanche pas trouvé d'ouvrage synthétique exhaustif sur les " marraines de guerre ", ce qui, sauf erreur, mériterait une enquête de grande ampleur.

29. Cf. pour les Allemandes, les travaux d'Ute Daniel et sa synthèse dans Hirschfeld / Krumeich / Renz, "Frauen ", p. 117-134. Et, pour la France, les travaux de Françoise Thébaud et sa synthèse dans Audouin-Rouzeau / Becker, t. 2, p. 110-127, ou encore Chantal Antier-Renaud, Les femmes dans la Grande Guerre, Saint Cloud, Soteca, 2011. 
